\title{
REPRODUTIBILIDADE DA CLASSIFICAÇÃO DA AO/ASIF PARA FRATURAS DOS OSSOS LONGOS NA CRIANÇA
}

\author{
REPRODUCIBILITY OF THE AO / ASIF \\ CLASSIFICATION FOR FRACTURES OF LONG BONES IN CHILDREN
} Gilberto Francisco Brandão', Luiz Eduardo Moreira Teixeira², Luiz Renato Drumond Américo ${ }^{3}$, Cláudio Beling Gonçalves
Soares $^{3}$, Leonardo Ornelas Caldas ${ }^{3}$, Ana Flávia Figueiredo Azevedo ${ }^{4}$, Lucas de Castro Bouchat ${ }^{5}$

\section{RESUMO}

Objetivo: Avaliar a reprodutibilidade intraobservador e interobservador da classificação proposta pelo grupo AO/ASIF, para as fraturas dos ossos longos em crianças. Método: Foram analisadas e classificadas por cinco avaliadores, 100 radiografias convencionais digitalizadas de fraturas dos ossos longos em crianças, utilizando a classificação alfanumérica proposta pelo grupo AO/ASIF. A força de concordância intraobservador (realizada com intervalo de 90 dias) e a interobservador foram avaliadas através do coeficiente Kappa. Resultados: O índice Kappa para observação intraobservador foi de 0,69 (grande concordância) e na análise interobservador o índice foi de 0,64 (grande concordância) na primeira avaliação e 0,61 (grande concordância) na segunda análise realizada 90 dias após a classificação inicial. Conclusão: A classificação proposta pelo grupo AO/ASIF mostrou grande concordância intra e interobservador, sendo útil para utilização nas fraturas dos ossos longos em crianças.

Descritores - Fraturas Ósseas; Fraturas Ósseas/classificação; Criança

\section{ABSTRACT}

Objective: To evaluate the interobserver and intraobserver reproducibility of the classification proposed by the AO/ ASIF group for long bone fractures in children. Methods: One hundred roentgenograms of long bone fractures in children were evaluated and classified according to the alphanumeric AO/ASIF classification by six observers. The strength of theintraobserver(performed with a 90-day interval) and interobserveragreementwere evaluated using the Kappa coefficient. Results: The intraobserver Kappa index was 0.69 (good agreement). The interobserver index was 0.64 (good agreement) in the first evaluation and 0.61 (goodagreement) in the second analysisperformed 90 days after the initial classification. Conclusion: The classification proposed by the AO/ASIF group for long bone fractures demonstrated good inter-and intraobserver agreement. This classification is useful for describing fractures of long bones in children.

Keywords - Fracture Bone; Fractures, Bones/classification; Child

\section{INTRODUÇÃO}

As classificações utilizadas em ortopedia têm como objetivo orientar o tratamento, prever o prognóstico e permitir a padronização, documentação e comparação entre os vários estudos científicos ${ }^{(1-3)}$. Para isso, a classificação deve ser simples, de fácil aplicação na prática clínica e reprodutível, com grande concordância entre os cirurgiões ${ }^{(3)}$.

Embora vários sistemas de classificação tenham sido descritos para as fraturas da criança, a maioria das classificações é direcionada para ossos específicos como a de Gartland ${ }^{(4)}$ para as fraturas distais do úmero ou

\footnotetext{
1 - Ortopedista Pediátrico do Hospital das Clínicas - UFMG e Hospital da Baleia. Membro Sociedade Brasileira de Ortopedia Pediátrica e SBOT.

2 - Professor Assistente do Departamento do Aparelho Locomotor da Faculdade de Medicina da Universidade Federal de Minas Gerais - UFMG.

3 - Médico Ortopedista Pediátrico do Hospital da Baleia; Membro Titular da Sociedade Brasileira de Ortopedia Pediátrica.

4 - Residente de Radiologia do Biocor Instituto - Belo Horizonte, MG.

5 - Residente de Ortopedia do Hospital das Clínicas - UFMG.
}

Trabalho realizado no Hospital das Clínicas da Universidade Federal de Minas Gerais e Instituto Mineiro de Ortopedia e Traumatologia

Correspondência: Instituto Mineiro de Ortopedia e Traumatologia. Avenida do Contorno 7.485, Santo Antônio - 30110-047 - Belo Horizonte - MG.

E-mail: ortopedia.pediatrica@yahoo.com.br 
para lesões fisárias como a de Salter e Harris ${ }^{(5)}$. Outras classificações são simplistas, generalistas, e carecem de comprovação científica ${ }^{(3)}$.

Recentemente, o grupo $\mathrm{AO}$, em cooperação com o International Working Group for Pediatric Traumatology (iAGKT), publicou uma classificação para as fraturas dos ossos longos na criança que combina a de Muller et al ${ }^{(6)}$ para adultos com descrições direcionadas ao esqueleto imaturo $^{(3)}$. Trata-se de um sistema alfanumérico que inclui o osso acometido, a localização e a gravidade, associado a peculiaridades do osso em crescimento.

O objetivo deste estudo é avaliar a reprodutibilidade da classificação proposta pelo grupo AO para as fraturas dos ossos longos em crianças e sua validade para aplicação clínica.

\section{MATERIAL E MÉTODOS}

O estudo foi realizado no Instituto Mineiro de Ortopedia e no Hospital das Clínicas da Universidade Federal de Minas Gerais (UFMG), após aprovação pelo comitê de ética de ambos os serviços.

Foram avaliadas 100 radiografias convencionais selecionadas a partir de exames realizados no atendimento inicial de pacientes com fraturas de ossos longos, incluindo o úmero $(\mathrm{n}=18)$, fêmur $(\mathrm{n}=25)$, ossos do antebraço $(n=32)$ e tíbia $(n=25)$. As imagens foram digitalizadas e ordenadas de maneira randomizada; a seleção não considerou a qualidade do exame realizado, sendo avaliadas sempre nas incidências em anteroposterior e lateral. Foram excluídos pacientes maiores de 16 anos ou que apresentavam linha fisária fechada e pacientes com lesões múltiplas.

A seleção dos examinadores foi realizada considerando a inclusão de médicos experientes no tratamento de fraturas de crianças, residentes e radiologistas. Foram incluídos cinco examinadores, sendo três ortopedistas pediátricos $(1,2,3)$, um médico residente em ortopedia e traumatologia (4) e um radiologista (5). Todos os examinadores tiveram acesso à classificação e foi permitido treinamento durante um período de 30 dias antes da análise. Os examinadores realizaram a avaliação das 100 imagens com tempo máximo de avaliação de duas horas, sendo que após 90 dias realizaram a segunda avaliação, novamente com a ordem das imagens modificada por randomização.

Após preenchimento, todos os resultados eram recolhidos e analisados com auxílio do software SPSS ${ }^{\circledR}$ versão 12.0 (Chicago, EUA), para determinação do co- eficiente Kappa, sendo que as concordâncias intraobservador e interobservador foram determinadas de acordo com a Tabela 1 .

Tabela 1 - Associação do coeficiente Kappa e a força de concordância.

\begin{tabular}{c|c}
\hline Coeficiente Kappa & Força da Concordância \\
\hline Menos de zero & Pobre \\
\hline $0,00-0,20$ & Desprezível \\
\hline $0,21-0,40$ & Leve \\
\hline $0,41-0,60$ & Moderada \\
\hline $0,61-080$ & Grande \\
\hline $0,81-1,00$ & Quase perfeita \\
\hline
\end{tabular}

\section{RESULTADOS}

Os examinadores completaram sua análise no tempo determinado, sendo que todas as imagens foram classificadas por todos, sem que alguma imagem ficasse sem classificação.

A análise intraobservador apresentou média global do coeficiente Kappa de 0,69 $\pm 0,11$, sendo que os resultados individuais e a comparação entre as leituras estão na Tabela 2, sendo que a maior concordância foi conseguida por um ortopedista pediátrico $(\kappa=0,83)$ e a menor por um radiologista $(\kappa=0,42)$.

Para a avaliação interobservador, encontramos índice Kappa médio de 0,64 para a primeira avaliação e de 0,61 para a segunda avaliação, determinando grande concordância entre avaliadores nas duas avaliações.

Tabela 2 - Coeficiente Kappa e concordância entre as leituras intraobservador.

\begin{tabular}{c|c|c}
\hline Comparação entre as leituras & Kappa & Concordância \\
\hline A1a $\times$ a1b & 0,83 & Quase perfeita \\
\hline A2a $\times$ a2b & 0,71 & Grande \\
\hline A3a $\times$ a3b & 0,79 & Grande \\
\hline A4a $\times$ a4b & 0,68 & Grande \\
\hline A5a $\times$ a5b & 0,42 & Moderada \\
\hline
\end{tabular}

Legenda: $\mathrm{A}$ - examinadores, $\mathrm{a}$ - primeira leitura, $\mathrm{b}$ - segunda leitura.

\section{DISCUSSÃO}

A classificação proposta pelo grupo AO é baseada na classificação de Muller et $a l^{(6)}$ para adultos que utiliza um modelo alfanumérico, determinando o osso fraturado, o local e a gravidade da lesão. Para ser aplicada ao esqueleto imaturo, foi acrescentado um código que indica características da fratura que são peculiares às 
crianças (child code), como lesões fisárias, deformações plásticas e avulsões apofisárias (Figura 1).

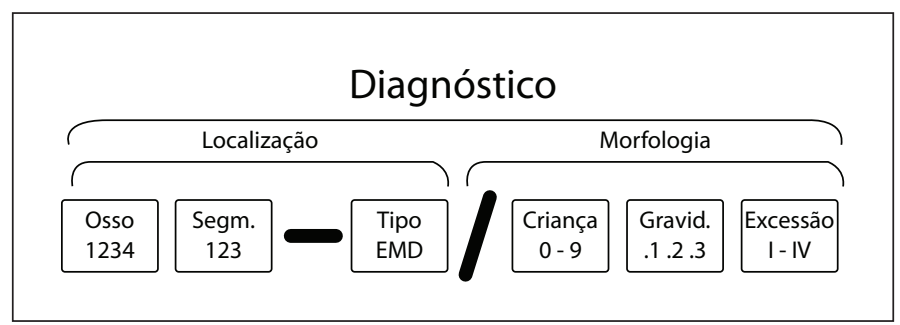

Figura 1 - Modelo alfanumérico da classificação AO para fraturas em ossos longos de crianças.

A classificação de uma fratura deve ser simples, reprodutível, permitir a comparação entre estudos científicos e apresentar alta concordância entre examinadores. De acordo com Audigé et al ${ }^{(1)}$, para alcançar esses objetivos, a classificação deve passar por três fases de pesquisa antes que seja validada para uso clínico. A primeira fase é o desenvolvimento da classificação por um grupo de médicos experientes no tratamento dessas fraturas e baseada em diversos estudos pilotos. Nesta fase, a concordância entre os cirurgiões deve ser superior a 90\%. Na segunda fase, a classificação é testada por examinadores de diferentes níveis de prática e conhecimento e a fase final é a sua aplicação clínica e documentação durante algum período de tempo para confirmar sua validade.

\section{REFERÊNCIAS}

1. Audigé $L$, Bhandari $M$, Kellam J. How reliable are reliability studies of fracture classifications? A systematic review of their methodologies. Acta Orthop Scand. 2004;75(3):184-94.

2. Garbuz DS, Masri BA, Esdaile J, Duncan CP. Classification systems in orthopaedics. J Am Acad Orthop Surg. 2002;10(4):290-7.

3. Slongo T, Audigé L, Schlickewei W, Clavert, J; Hunter, J Development and validation of the $\mathrm{AO}$ paediatric comprehensive classification of long-bone fractures.
Nosso estudo avaliou a validade da classificação por examinadores com vários graus de experiência no tratamento das fraturas da criança e na avaliação radiográfica destas fraturas (segunda fase de validação). Este estudo mostrou que a classificação apresentou grande concordância intra e interobservador. Apesar desta concordância, não conseguimos atingir um coeficiente Kappa superior a 0,80, que seria recomendável para esta fase. $\mathrm{O}$ menor índice do radiologista sugere que, além da experiência no diagnóstico, a prática no tratamento destas fraturas auxilia na familiarização com a classificação.

Acreditamos que a concordância não foi maior em decorrência da complexidade da classificação. Apesar de incluir os vários tipos de fratura da criança, sua memorização é difícil, o que pode seu uso de rotina.

Outros estudos semelhantes devem ser realizados para avaliar efeitos sociais, culturais e de qualidade de imagens que possam influenciar na prática ortopédica e torná-la útil ao cirurgião ortopedista.

\section{CONCLUSÃO}

A reprodutibilidade da classificação da $\mathrm{AO}$ para fraturas dos ossos longos da criança mostrou-se com grande concordância, tanto para a avaliação interobservador, quanto para a avaliação intraobservador.

J Pediatr Orthop. 2006;26(1):43-9.

4. Gartland JJ. Management of supracondylar fractures of the humerus in children. Surg Gynecol Obstet. 1959;109(2):145-54.

5. Salter RB, Harris WR. Injuries involving the epiphyseal plate. J Bone Joint Surg Am. 1963;45:587-622.

6. Muller ME, Nazarian S, Koch P. The comprehensive classification of fractures of long bones. Berlin: Springer-Verlag; 1990. 